Original

\title{
Laboratory evaluation of dentin tubule occlusion after use of dentifrices containing stannous fluoride
}

\author{
Toshiki Takamizawa',2), Akimasa Tsujimoto',2), Ryo Ishii1), Maho Ujiie1), \\ Mami Kawazu' ${ }^{1}$, Toshie Hidari1), Takayuki Suzuki1), and Masashi Miyazaki ${ }^{1,2)}$ \\ 1)Department of Operative Dentistry, Nihon University School of Dentistry, Tokyo, Japan \\ 2)Dental Research Center, Division of Biomaterial Science, Nihon University School of Dentistry, \\ Tokyo, Japan
}

(Received May 23, 2018; Accepted August 13, 2018)

\begin{abstract}
The purpose of this study was to examine the effect of desensitizing dentifrices containing stannous fluoride $\left(\mathrm{SnF}_{2}\right)$ on dentinal tubule occlusion. Two experimental dentifrices with the same ingredients but different $\mathrm{SnF}_{2}$ concentrations (Group II, $0.4 \%$ w/w; Group III, $0.454 \%$ w/w) were used; distilled water was used in the control group (Group I). Third molars were collected from Japanese and American dental patients. The crowns were removed and sectioned to obtain dentin discs, which were further cut into quarters. Thirty-six specimens each from Japanese and American patients were divided into three sets $(n=12$ each) and assigned to each of the three treatment groups. The specimens were brushed for $10 \mathrm{~s}$ twice per day for $\mathbf{4}$ days. After treatment, the discs were observed by scanning electron microscopy, and the extent of dentinal tubule occlusion in the images was expressed on a five-point categorical scale. Group II and III specimens from Japanese and American patients showed greater dentinal tube occlusion than those from Group I, but the differences were not statistically significant. The present results suggest that both $\mathrm{SnF}_{2}$ concentrations mitigate dentin hypersensitivity, regardless of patient ethnicity.
\end{abstract}

Correspondence to Dr. Toshiki Takamizawa, Department of Operative Dentistry, Nihon University School of Dentistry, 1-8-13 Kanda-Surugadai, Chiyoda-ku, Tokyo 101-8310, Japan

Fax:+81332198347 E-mail: takamizawa.toshiki@nihon-u.ac.jp

Color figures can be viewed in the online issue at J-STAGE.

doi.org/10.2334/josnusd.18-0176

DN/JST.JSTAGE/josnusd/18-0176
Keywords: dentin hypersensitivity; stannous fluoride; dentifrice; dentinal tubule occlusion; four-day occlusion method.

\section{Introduction}

Dentin hypersensitivity - short, sharp pain induced by external stimulation - is a common oral health problem $(1,2)$. The hydrodynamic theory of the mechanism of dentin hypersensitivity is widely accepted $(3,4)$. External stimuli such as thermal, tactile, chemical, or osmotic pressure provoke movement of intratubular dentinal fluids in exposed dentin because of the absence of cementum after gingival recession $(5,6)$. The symptoms of dentin hypersensitivity can be mitigated by modifying the surface of exposed dentin and occluding dentinal tubules (7). Several approaches, such as application of chemical agents or substances similar to hydroxyapatite, filling or sealing of cavities with resin-based materials or glass-ionomer cements, and use of gingival grafts, have been recommended to reduce hypersensitivity in dental clinics (8-10). Chemical agents such as potassium nitrate and potassium chloride are thought to suppress pain by penetrating dentinal tubules and elevating potassium ion levels (8). In addition, organic and inorganic chemicals and their compounds have been used to block dentinal fluid flow, thereby reducing hydraulic conductance across dentinal tubules (8-10). However, no single method or desensitizing agent is ideal for the treatment of hypersensitivity. Therefore, a treatment strategy based on hypersensitivity stage should be developed and implemented.

Use of dentifrices that include a chemical compound 
Table 1 Materials used in the study

\begin{tabular}{lcll}
\hline Group & $\begin{array}{c}\text { Experimental dentifrice } \\
\text { (Lot No.) }\end{array}$ & \multicolumn{1}{c}{ Main components } & Manufacturer \\
\hline I & - & Distilled water & - \\
II & $(1470 \mathrm{~B} 302)$ & $\begin{array}{l}0.4 \% \text { w/w stannous fluoride, glycerin, PEG-8, } \\
\text { hydrated silica, pentasodium triphosphate, } \\
\text { sodium lauryl sulphate, flavor, } \\
\text { titanium dioxide, polyacrylic acid, } \\
\text { cocamidopropyl betaine, sodium saccharin }\end{array}$ & $\begin{array}{l}\text { GlaxoSmithKline } \\
\text { Consumer Healthcare, } \\
\text { Middlesex, UK }\end{array}$ \\
& $\begin{array}{l}\text { 0.454\% w/w stannous fluoride, glycerin, PEG-8, } \\
\text { hydrated silica, pentasodium triphosphate, } \\
\text { sodium lauryl sulphate, flavor, } \\
\text { titanium dioxide, polyacrylic acid, } \\
\text { cocamidopropyl betaine, sodium saccharin }\end{array}$ & $\begin{array}{l}\text { GlaxoSmithKline } \\
\text { Consumer Healthcare, } \\
\end{array}$ \\
& (1470B283) & Middlesex, UK
\end{tabular}

PEG-8: polyethylene glycol 400.

to occlude dentinal tubules or desensitize the peripheral sensory nerves in the dental pulp is the treatment of choice because of its low cost and the easy availability of these substances in daily tooth brushing (8). Potassium oxalate, aluminum lactate, strontium chloride, and complex fluorides are commonly included in dentifrices, to mitigate pain (9). However, these dentifrices occasionally fail to reduce pain or may lose their effect on dentin hypersensitivity over time. Therefore, a more reliable and longer-lasting dentin-desensitizing dentifrice is needed.

Dentifrices containing stannous fluoride $\left(\mathrm{SnF}_{2}\right)$ have anti-caries and anti-gingivitis effects and have been used for over 50 years (11). Moreover, previous studies reported that these dentifrices may reduce enamel erosion (12-14), breath odor (15), and dentin hypersensitivity (16-22). Nevertheless, concerns have been expressed regarding the formulation of these dentifrices, particularly with respect to the stability of stannous ions versus bioavailability, the tendency to enhance extrinsic dental stains, and the unpleasant taste (23). To prevent degradation of $\mathrm{SnF}_{2}$ into insoluble and inactive stannic hydroxides and oxides and mitigate dental staining, an anhydrous $\mathrm{SnF}_{2}$ formulation that includes the chelating agent sodium tripolyphosphate was developed (21). However, the effect of anhydrous $\mathrm{SnF}_{2}$-containing dentifrices on dentin hypersensitivity is not clear.

The present study used a 4-day occlusion method to examine the effect of a newly formulated dentindesensitizing dentifrice including $\mathrm{SnF}_{2}$. The effect was evaluated by using a categorical occlusion scale for dentinal tubules and scanning electron microscopy (SEM) of samples collected from patients who were asked to brush their teeth with this dentifrice twice daily for up to 4 days. In addition, the teeth of Japanese and American patients were compared to investigate the effect of patient ethnicity on the ability of the dentifrice to occlude dentinal tubules. The null hypotheses tested were that the effectiveness of experimental desensitizing dentifrices is not influenced by $\mathrm{SnF}_{2}$ concentration and that the status of occluded dentinal tubules would not differ between Japanese and American patients.

\section{Study materials}

\section{Materials and Methods}

Two experimental dentifrices with the same ingredients but different percentages of $\mathrm{SnF}_{2}$ (Group II, $0.4 \% \mathrm{w} / \mathrm{w}$ $\mathrm{SnF}_{2}$; Group III, $0.454 \% \mathrm{w} / \mathrm{w} \mathrm{SnF}_{2}$ ) were used (Table 1). Distilled water was used for the control group (Group I).

\section{Collection of human teeth}

To investigate the influence of patient ethnicity on occlusion of dentinal tubules by the dentifrices, third molars were collected from Japanese and American dental patients. Caries-free third molars extracted for orthodontic or other medical/dental reasons were obtained from Japanese patients who visited the dental hospital at the Nihon University School of Dentistry. Informed consent for the study was obtained from all patients. In addition, unerupted third molars from American patients (with ethical committee approval from GlaxoSmithKline) were sourced through an appropriate contracted supplier of GlaxoSmithKline Consumer Health. The study protocol was reviewed and approved by the Ethics Committee for Human Studies of the Nihon University School of Dentistry (2015-03). Partially and fully erupted molars were not included in this study. Unerupted third molars of regular size and shape were selected, and teeth with any signs of cracking in the enamel or across the base were excluded. Specimens for which the extraction procedure caused slight scuffing of the enamel were included. After extraction, soft tissue attached to the root was immediately removed with hand instruments, 
and the tooth was then immersed in distilled water for 6 h. The extracted teeth were stored frozen $\left(-20^{\circ} \mathrm{C}\right)$ until further experiments.

\section{Specimen preparation}

The crowns of the teeth were sectioned perpendicular to the long axis of the roots, just above the enamel-dentin junction, with a diamond disc saw (Isomet 1000; Buehler Ltd, Lake Bluff, IL, USA) and copious water cooling, to remove most of the crown. The specimens were rinsed with distilled water to remove any debris and stored in $0.1 \% \mathrm{w} / \mathrm{w}$ thymol solution in a sealed container. Dentin disc specimens free from cracks, staining, and other imperfections were prepared as follows: the removed crowns were sectioned parallel to the cut surface with a diamond saw to obtain a dentin disc (thickness, 1.0-1.5 $\mathrm{mm})$. Only one disc could be cut from each crown. Each dentin disc specimen was then cut into quarters with a diamond bur. One quarter from each dentin disc was placed in a 25-mm mold, with the crown side of the specimen facing down, and covered with acrylic resin to a depth of 10 mm (Resin tray II; Shofu Inc., Kyoto, Japan). After the resin had hardened at room temperature and atmospheric pressure, the specimen was removed from the mold, and the dentin face was polished sequentially with 800-, 1200-, and 2,500-grit silicon carbide papers (Struers Inc., Cleveland, OH, USA), and finally with a $0.25-\mu \mathrm{m}$ diamond polishing suspension (DP-Paste; Struers, Ballerup, Denmark), to a mirror finish. The specimens were cleaned in an ultrasonic bath (Quantrex 310; L\&R Mfg., Co., Kearny, NJ, USA) for $120 \mathrm{~s}$ with distilled water to remove any occluding material. Finally, the specimens were rinsed with distilled water for 60 s. One quarter of each specimen was observed with a confocal laser scanning microscope (VK-8700; Keyence Corp., Osaka, Japan) at $\times 1,000$ magnification, to verify specimen integrity and tubule density and patency. If the specimen did not appear to have consistently circular, patent tubules, the initial and remaining three quarters of the dentin specimen were discarded. Otherwise, the remaining three dentin quarters were placed into separate 25-mm molds and polished in the manner described above. Polished dentin specimens were sonicated for 120 $\mathrm{s}$ in distilled water to remove any occluding material and then rinsed for $60 \mathrm{~s}$ in distilled water. Specimen integrity and tubule density and patency were verified with a confocal laser scanning microscope. When the characteristics of all four quarters were deemed satisfactory, the specimen was included in the study, and each of the three remaining quarters was allocated to one treatment. The specimens were prepared and verified until 12 acceptable
Table 2 Components of artificial saliva

\begin{tabular}{ll}
\hline Potassium chloride & $30 \mathrm{mM}$ \\
Calcium chloride di-hydrate & $3 \mathrm{mM}$ \\
Potassium di-hydrogen orthophosphate & $10 \mathrm{mM}$ \\
Sodium chloride & $13 \mathrm{mM}$ \\
Type II Porcine stomach mucin (M2378 Sigma) & $0.22 \% \mathrm{w} / \mathrm{w}$ \\
Sodium azide as preservative & $0.02 \% \mathrm{w} / \mathrm{w}$ \\
\hline
\end{tabular}

discs from both Japanese and American patients had been prepared.

A total of 36 dentin specimens from each country progressed through to treatment application, excluding gold-sputtered specimens. The specimens from each country were divided into three sets of 12 each, which ensured that one specimen from each tooth was present in each treatment group. A set of 12 dentin specimens was assigned to each of the three treatment groups, for each source of dentin.

\section{Treatment procedures (4-day occlusion method)}

The specimens were treated twice daily for up to 4 days. They were then disinfected using Corsodyl mouth rinse (Concool F; Weltec Corp., Osaka, Japan) for 10 $\mathrm{s}$, followed by rinsing with distilled water for $10 \mathrm{~s}$. All specimens from one country of origin were immersed in a beaker containing $50 \mathrm{~mL}$ of artificial saliva (Table 2) for $60 \mathrm{~min}$ with no stirring. After $60 \mathrm{~min}$, the first brushing was performed for each group. For dentin disc treatment, an electric toothbrush (Braun Oral-B 5000 with wireless smartguide, Oral-B FlossAction head; Procter \& Gamble, Cincinnati, OH, USA) was used in daily clean mode (Fig. 1). Each specimen was brushed after wetting the brush head with distilled water and applying $1.1 \pm 0.1 \mathrm{~g}$ of test dentifrice to the brush head. Brushing was performed uniformly for $10 \mathrm{~s}$ across a resin plug containing three dentin specimens assigned to a single treatment group. An equivalent brushing force of approximately $100 \mathrm{~g}$ was used, as measured with an electronic scale (AE163, Mettler-Toledo International Inc., Tokyo, Japan). The specimens were left for $30 \mathrm{~s}$ before being gently rinsed in distilled water for $10 \mathrm{~s}$, thus ensuring that all visible paste was removed. The brush head was cleaned in distilled water with an ultrasonic bath, and new paste was applied for each separate treatment. The specimens were then returned to $50 \mathrm{~mL}$ of fresh artificial saliva and stored for at least $4 \mathrm{~h}$.

The second brushing was carried out as described above. At the end of days 1-3, the specimens were placed in a sealed pot on tissue paper wetted with distilled water for storage overnight at $5 \pm 2^{\circ} \mathrm{C}$, for treatment the next 


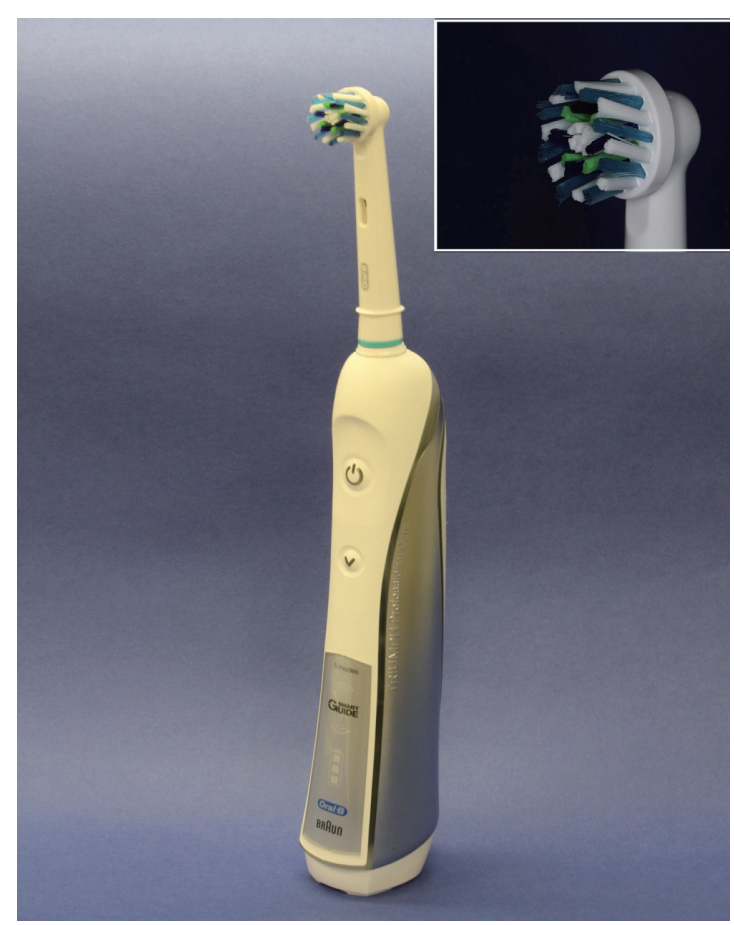

Fig. 1 Electric toothbrush used in this study.

day. At the end of day 4, the specimens were removed, washed with distilled water, and allowed to dry overnight at room temperature and atmospheric pressure in preparation for SEM.

\section{Scanning electron microscopy (SEM)}

All dentin discs were observed after treatment by fieldemission SEM (ERA-8800FE; Elionix Inc., Tokyo, Japan). The specimens were coated with a thin film of gold $(\mathrm{Au})$ in a Quick Coater vacuum evaporator (Type SC-701; Sanyu Electron Co., Tokyo, Japan). To ascertain the uniformity of the dentin profile, five predesignated areas of the dentin were imaged in each dentin section. At least three representative SEM micrographs were acquired at $\times 1,500$ magnification (three images per disc quarter). Observations were performed at an operating voltage of $10 \mathrm{kV}$.

\section{Evaluation of dentinal tubule occlusion}

Each SEM image was assessed for the extent of dentinal tubule occlusion, as expressed on a five-point categorical scale (Fig. 2A-2E), by a single examiner from the Department of Operative Dentistry, Nihon University School of Dentistry. Before the study began, the examiner was calibrated against a set of standard micrographs encompassing the entire grading scale. The examiner was blinded to the type of treatment and did not observe the images being taken.
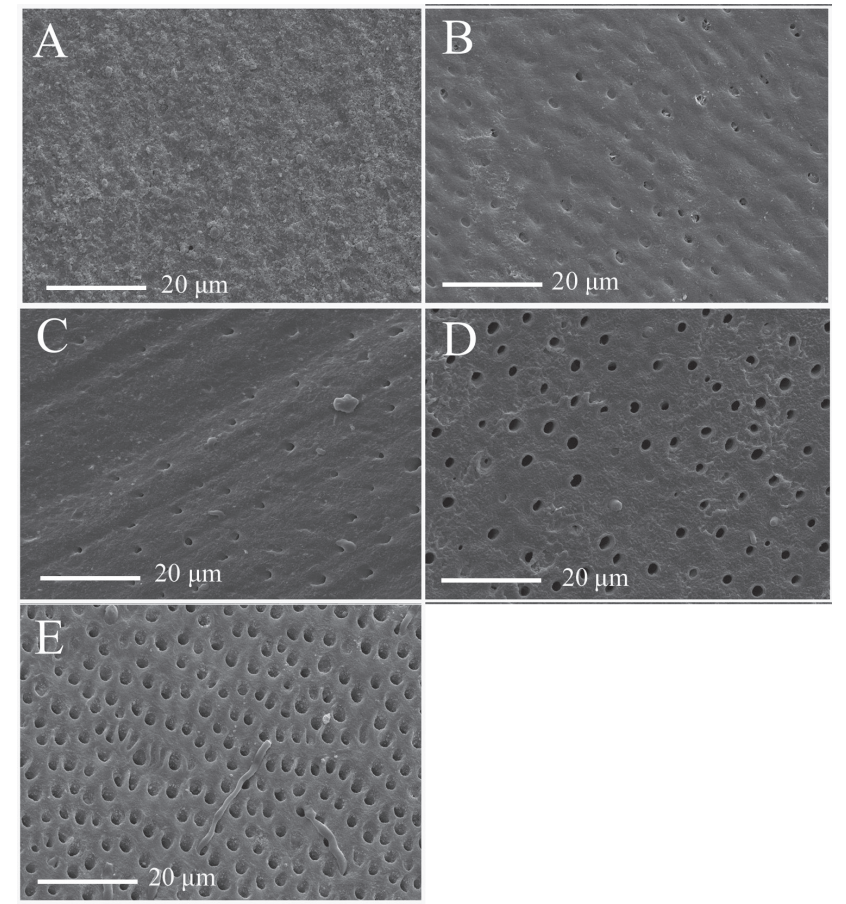

Fig. 2 Dentinal tubule occlusion was evaluated by using a fivepoint categorical scale. 2A. 1, occluded. 2B. 2, mostly occluded. 2C. 3, equal. 2D. 4, mostly unoccluded. 2E. 5, unoccluded.

\section{Energy-dispersive X-ray spectroscopy (EDX)}

The elements present in representative dentin surfaces from each group were analyzed with SEM/EDX (GENESIS 2000; EDAX Ametek Co., Tokyo, Japan) at $20 \mathrm{kV}$ and $\times 1,500$ magnification. Specimens brushed with distilled water (controls) were also analyzed. Measurements were taken perpendicular to the prepared dentin specimens. The elemental content (wt\%) of the $\mathrm{Au}$-coated surface was measured at three regions, as close as possible to the center, in five specimens from each group, and the mean value was determined for each group. The analysis was performed with ZAF correction (atomic number, absorption, and fluorescence), based on standard-less correction (24).

\section{Statistical analysis}

Treatment differences were analyzed by using the Kruskal-Wallis test followed by the Steel-Dwass test at a significance level of 0.05 , and differences in relation to national origin were analyzed by using the Mann-Whitney $U$ test with Bonferroni correction at a significance level of 0.05 . In SEM/EDX analysis, data from each group and each detected element were analyzed with the Kruskal-Wallis test followed by the Steel-Dwass test at a significance level of 0.05 . All statistical analysis was done with the Sigma Plot, ver. 11.0, software program (SPSS Inc., Chicago, IL, USA). 


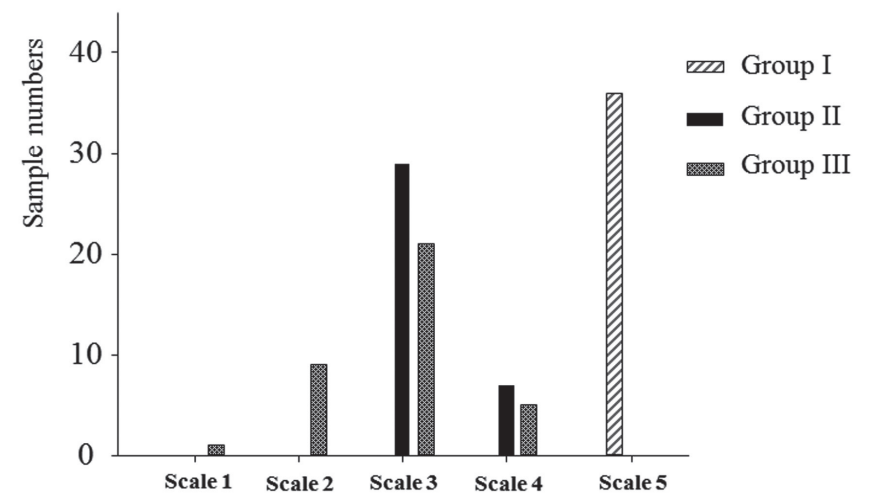

Fig. 3 Scoring of dentinal tubule occlusion in Japanese specimens.

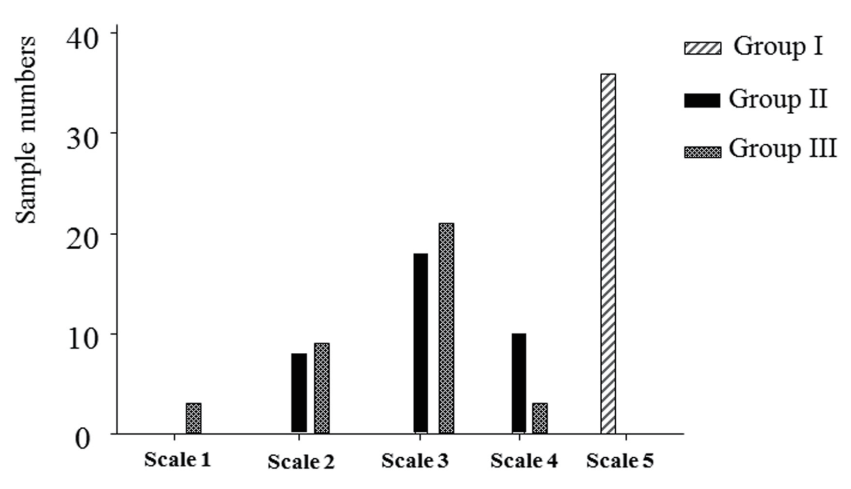

Fig. 4 Scoring of dentinal tubule occlusion in American specimens.

Table 3 Evaluation of dentinal tubule occlusion using a five-point categorical scale

\begin{tabular}{lccc}
\hline Ethnicity & $\begin{array}{c}\text { Group I } \\
\text { Control }\end{array}$ & $\begin{array}{c}\text { Group II } \\
0.4 \% \mathrm{w} / \mathrm{w} \mathrm{SnF}_{2}\end{array}$ & $\begin{array}{c}\text { Group III } \\
0.454 \% \mathrm{w} / \mathrm{w} \mathrm{SnF}_{2}\end{array}$ \\
\hline Japanese & $5.0(0)^{\mathrm{a}, \mathrm{A}}$ & $3.19(0.36)^{\mathrm{a}, \mathrm{B}}$ & $2.86(0.69)^{\mathrm{a}, \mathrm{B}}$ \\
American & $5.0(0)^{\mathrm{a}, \mathrm{A}}$ & $3.06(0.70)^{\mathrm{a}, \mathrm{B}}$ & $2.67(0.76)^{\mathrm{a}, \mathrm{B}}$ \\
\hline Presence of identical lowercase letters in a column indicates no difference at a 5\% significance level. \\
Presence of identical uppercase letters in a row indicates no difference at a 5\% significance level.
\end{tabular}

\section{Results}

\section{Evaluation of dentinal tubule occlusion}

Figures 3 and 4 show the categorization of specimens, by group. All Group I specimens (regardless of national origin) were scored as 5 (unoccluded dentinal tubules). In Group II, all specimens from Japanese patients were scored as 3 or 4 , and all specimens from American patients were scored as 2, 3, or 4. In Group III, specimens from Japanese and American patients were scored as 1 through 4. In Groups II and III, the numbers of specimens with a score of 3 far exceeded the numbers of specimens with other scores, regardless of patient ethnicity.

Table 3 shows the results of the Kruskal-Wallis test followed by the Steel-Dwass test, for comparison of treatment, and the results of the Mann-Whitney $U$ test with Bonferroni correction, for comparison of specimen origin. Although values from Groups II and III did not significantly differ $(P>0.05)$ for Japanese specimens, dentinal tube occlusion was significantly greater in these groups than in Group I $(P<0.05)$. The trend was similar for American specimens, i.e., dentinal tube occlusion was significantly greater in Groups II and III than in Group I $(P<0.05)$. In addition, the results did not significantly differ in relation to specimen origin $(P>0.05)$.

\section{SEM observation}

Regardless of patient ethnicity, all dentinal tubules in the initial dentin specimens were completely opened, and smear plugs were absent after mirror polishing and ultrasonic cleaning for $120 \mathrm{~s}$, indicating that the dentinal tubules were free from debris (Figs. 5A, 6A). The morphological appearance of specimens in treatment Group I (without dentifrice) was similar to that of the initial specimens from both ethnic groups (Figs. 5B, 6B). No deposit or debris in the intertubular dentin or dentinal tubules was noted. In contrast, the morphological characteristics of American and Japanese specimens in treatment Groups II and III differed from those in Group I and the initial dentin specimens (Figs. 5CD, 6CD). Some deposits derived from the dentifrice were observed in the intertubular dentin and dentinal tubules. In particular, the dentinal tubules in Group III appeared to be more occluded than those in Group II (Figs. 5D, 6D). Under higher magnification, aggregated small particles in the dentinal tubules and granular deposits on the dentin surface were observed in specimens from Group III.

\section{Energy-dispersive X-ray microanalysis}

The elements present in representative dentin surfaces from each group are shown in Table 4. Si and Sn were detected in Groups II and III but not in Group I. No significant differences in elemental profile were noted between Groups II and III, regardless of specimen origin. The control specimens from Japanese and American patients had the same elements and similar concentrations of those elements.

\section{Discussion}

To overcome the drawbacks of earlier dentifrices containing $\mathrm{SnF}_{2}$, advanced technologies have been devel- 


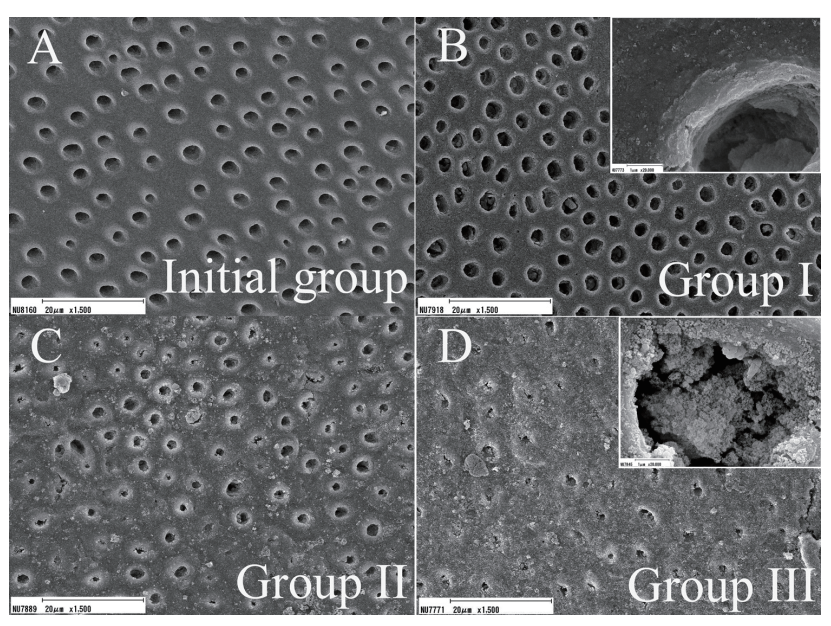

Fig. 5 Representative SEM images of Japanese dentin specimens showing morphological differences in relation to treatment. 5A. Initial specimen of dentin $(\times 1,500)$. 5B. Treatment Group I $(\times 1,500$ and $\times 20,000)$. 5C. Treatment Group II $(\times 1,500)$. 5D. Treatment Group III $(\times 1,500$ and $\times 20,000)$.

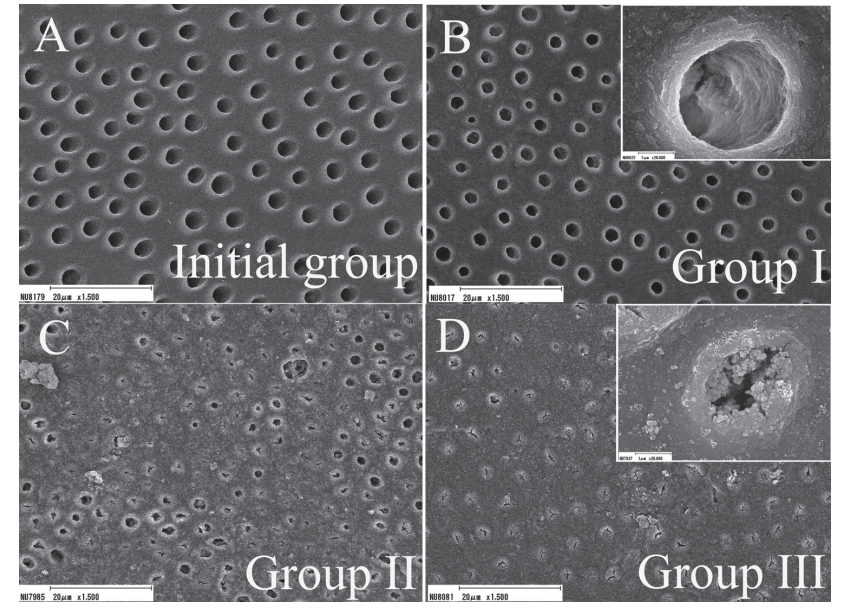

Fig. 6 Representative SEM images of American dentin specimens showing morphological differences in relation to treatment. 6A. Initial specimen of dentin $(\times 1,500)$. 6B. Treatment Group I $(\times 1,500$ and $\times 20,000)$. 6C. Treatment Group II $(\times 1,500)$. 6D. Treatment Group III $(\times 1,500$ and $\times 20,000)$.

Table 4 Effect of dentifrices on elemental composition (wt\%) on the dentin surface

\begin{tabular}{lccccc}
\hline \multicolumn{2}{c}{ Group I } & \multicolumn{2}{c}{ Group II } & \multicolumn{2}{c}{ Group III } \\
Japanese & American & Japanese & American & Japanese & American \\
\hline $\begin{array}{l}\text { Carbon } \\
\begin{array}{l}21.6(2.6)^{\mathrm{B}} \\
\text { Nitrogen }\end{array}\end{array}$ & $24.6(0.9)^{\mathrm{AB}}$ & $25.7(0.8)^{\mathrm{A}}$ & $21.9(3.2)^{\mathrm{B}}$ & $22.9(2.9)^{\mathrm{AB}}$ & $22.3(2.3)^{\mathrm{B}}$ \\
$\begin{array}{l}13.2(1.0)^{\mathrm{AB}} \\
\text { Oxygen }\end{array}$ & $14.0(0.9)^{\mathrm{AB}}$ & $14.3(0.8)^{\mathrm{A}}$ & $12.0(0.8)^{\mathrm{B}}$ & $12.3(0.9)^{\mathrm{B}}$ & $13.0(0.8)^{\mathrm{B}}$ \\
$\begin{array}{l}33.1(1.3)^{\mathrm{A}} \\
\text { Sodium }\end{array}$ & $32.1(1.2)^{\mathrm{A}}$ & $33.1(3.8)^{\mathrm{A}}$ & $30.1(1.8)^{\mathrm{A}}$ & $30.3(3.9)^{\mathrm{A}}$ & $32.1(1.5)^{\mathrm{A}}$ \\
$\begin{array}{l}0.7(0.2)^{\mathrm{A}} \\
\text { Magnesium }\end{array}$ & $0.6(0.1)^{\mathrm{A}}$ & $0.6(0.1)^{\mathrm{A}}$ & $0.7(0.1)^{\mathrm{A}}$ & $0.6(0.1)^{\mathrm{A}}$ & $0.7(0.1)^{\mathrm{A}}$ \\
$\begin{array}{l}0.4(0.1)^{\mathrm{A}} \\
\text { Silicon }\end{array}$ & $0.3(0.1)^{\mathrm{A}}$ & $0.4(0.1)^{\mathrm{A}}$ & $0.3(0.1)^{\mathrm{A}}$ & $0.3(0.1)^{\mathrm{A}}$ & $0.4(0.1)^{\mathrm{A}}$ \\
$\begin{array}{l}0.7(0.1)^{\mathrm{A}} \\
\text { Phosphorus }\end{array}$ & $\mathrm{NA}$ & $\mathrm{NA}$ & $0.7(0.1)^{\mathrm{A}}$ & $0.8(0.1)^{\mathrm{A}}$ & $0.8(0.1)^{\mathrm{A}}$ \\
$\begin{array}{l}11.6(0.6)^{\mathrm{A}} \\
\text { Tin }\end{array}$ & $10.8(0.9)^{\mathrm{A}}$ & $10.4(0.7)^{\mathrm{A}}$ & $11.8(2.3)^{\mathrm{A}}$ & $12.0(2.1)^{\mathrm{A}}$ & $11.9(2.0)^{\mathrm{A}}$ \\
$\begin{array}{l}1.8(0.3)^{\mathrm{A}} \\
\text { Calcium }\end{array}$ & $\mathrm{NA}$ & $\mathrm{NA}$ & $1.8(0.5)^{\mathrm{A}}$ & $1.7(0.4)^{\mathrm{A}}$ & $2.0(0.5)^{\mathrm{A}}$ \\
$17.6(1.1)^{\mathrm{A}}$ & $16.0(3.2)^{\mathrm{A}}$ & $15.7(3.3)^{\mathrm{A}}$ & $18.0(1.5)^{\mathrm{A}}$ & $17.8(1.0)^{\mathrm{A}}$ & $17.5(1.2)^{\mathrm{A}}$ \\
\hline
\end{tabular}

$n=10$, mean (SD) in wt $\%$.

Presence of identical uppercase letter in rows indicates no difference at a $5 \%$ significance level.

Values in parentheses are standard deviations.

oped to stabilize the compound and reduce tooth staining $(21,25)$. These technologies have led to a re-evaluation of the use of $\mathrm{SnF}_{2}$ to reduce caries, gingivitis, and enamel erosion and mitigate dentin hypersensitivity (12-22). Several studies around the world have investigated advanced $\mathrm{SnF}_{2}$-containing dentifrices (12-22). A recent 8 -week clinical trial of dentifrices containing anhydrous $0.454 \% \mathrm{w} / \mathrm{w} \mathrm{SnF}_{2}$ showed a significant $(>20 \%)$ reduction in sensitivity as compared with a control dentifrice containing 1,000 ppm sodium fluoride (19). Another clinical trial reported a statistically significant reduction in dentin hypersensitivity with a $0.454 \% \mathrm{w} / \mathrm{w}$ stabilized $\mathrm{SnF}_{2}$ dentifrice as compared with a negative control dentifrice. The desensitizing effect increased in magnitude with continued use (20).

The present results show that a $\mathrm{SnF}_{2}$ dentifrice (Groups II and III) induced significantly greater dentinal tube occlusion than did distilled water (Group I). The $\mathrm{SnF}_{2}$ desensitizing mechanism is attributable to formation of insoluble stannous compounds due to rapid oxidization $\left(\mathrm{Sn}^{2+}\right.$ to $\left.\mathrm{Sn}^{4+}\right)$ and hydrolysis of $\mathrm{SnF}_{2}$ in the presence of water or saliva (19). These compounds contribute to the formation of a layer over the dentin and reduce its permeability by creating precipitates within the dentinal tubules (26). Using an ultrasonic device, Endo et al. investigated the effect of a calcium phosphate desensitizer on dentin 
hypersensitivity and found that sonic velocity was significantly higher for the desensitizer-applied specimens than for negative and positive control specimens (27). They suggested that this higher sonic velocity might account for the small precipitates inside the dentinal tubules and the presence of a layer of granular precipitate on the dentin surface, and that occlusion of dentinal tubules might reduce dentinal fluid movement (27). The present SEM images from Groups II and III are consistent with these earlier findings and revealed deposits in intertubular dentin and dentinal tubules. The aggregates of small particles were presumed to be $\mathrm{SnF}_{2}$ compounds on the basis of detection of tin in EDX analysis.

The most common $\mathrm{SnF}_{2}$ concentration in dentifrices is $0.454 \% \mathrm{w} / \mathrm{w}$; however, $0.4 \% \mathrm{w} / \mathrm{w} \mathrm{SnF}_{2}$ has long been widely used as a topically applied gel. Although the Japanese Ministry of Health, Labor and Welfare began permitting the use of dentifrices containing up to 1,500 ppm fluoride in 2017, almost all commercially available dentifrices in Japan contain less than 1,000 ppm fluoride. However, it remains to be determined if dentifrices containing $0.454 \% \mathrm{w} / \mathrm{w}$ and $0.4 \% \mathrm{w} / \mathrm{w} \mathrm{SnF}_{2}$ differ in relation to dentinal tubule occlusion. The present results showed no significant difference in dentinal tubule occlusion between these $\mathrm{SnF}_{2}$ concentrations. Hence, the first null hypothesis - that the effectiveness of an experimental desensitizing dentifrice would not be influenced by the concentration of $\mathrm{SnF}_{2}$ - was not rejected. Dentifrices containing $0.454 \% \mathrm{w} / \mathrm{w}$ or $0.4 \% \mathrm{w} / \mathrm{w} \mathrm{SnF}_{2}$ may be used to mitigate dentin hypersensitivity.

Dentinal tubule occlusion did not significantly differ in relation to specimen origin under any of the treatment conditions. Hence, the second null hypothesis - that the status of occluded dentinal tubules would not differ between Japanese and American teeth - was not rejected. Many studies have investigated anatomical and genetic differences in teeth among populations (28-32). Previous studies showed that the crown dimensions of permanent teeth varied in relation to nationality, $(28,31)$ owing to genetic and environmental differences (31). A study of morphological differences in root dentin in four ethnic groups reported significant ethnic differences in sclerotic dentin proportion and tooth location (29). These reports suggest that the diameter and number of dentinal tubules might differ among populations. However, the present SEM images showed no clear morphological differences between Japanese and American specimens in the diameter or number of dentinal tubules. Furthermore, the elemental composition of the dentin substrate did not substantially differ by ethnicity in EDX analysis.

From a public-health perspective, daily tooth brushing with a dentifrice is undoubtedly the most common way to successfully prevent oral disease and maintain oral health (33). Tooth erosion related to wear can result in diminished esthetic appearance, functional occlusion, dentin hypersensitivity, and tooth fracture. Progression of root caries is caused by dentin exposure with gingival recession and poor oral hygiene and is common among elderly adults, because of their decreased saliva secretion. In addition to mitigating dentin hypersensitivity, dentifrices containing $\mathrm{SnF}_{2}$ will likely slow progression of erosion and root caries because they induce remineralization and have anti-plaque and anti-gingivitis properties.

The present results suggest that dentinal tubule occlusion was significantly greater for dentifrices containing $\mathrm{SnF}_{2}$ than in controls. In addition, dentinal tubule occlusion was greater for the dentifrice with a higher $\mathrm{SnF}_{2}$ concentration than for that with a lower concentration, although the difference was not significant. No significant difference in occlusion between Japanese and American specimens was noted after any of the present treatment methods. In conclusion, these results suggest that $0.454 \% \mathrm{w} / \mathrm{w}$ and $0.4 \% \mathrm{w} / \mathrm{w} \mathrm{SnF}_{2}$ are effective in mitigating dentin hypersensitivity, regardless of patient ethnicity.

\section{Acknowledgments}

This work was supported in part by a Grant-in-Aid for Scientific Research (C) (16K11565 and 17K11716) and a Grant-in-Aid for Young Scientists (B) (17K17141 and 17K17142) from the Japan Society for the Promotion of Science. This project was also supported in part by the Sato Fund and by a grant from the Dental Research Center of the Nihon University School of Dentistry, Japan.

\section{Conflict of interest}

This study was funded by GlaxoSmithKline. The sponsor had no control over the interpretation, writing, or publication of this work.

\section{References}

1. Holland GR, Närhi MN, Addy M, Gangarosa L, Orchardson R (1997) Guidelines for the design and conduct of clinical trials on dentine hypersensitivity. J Clin Periodontol 24, 808-813.

2. Gillam DG, Orchardson R (2006) Advances in the treatment of root sensitivity: mechanisms and treatment principles. Endod Topics 13, 13-33.

3. Brännström M, Linden L, Aström A (1967) The hydrodynamics of dental tubule and pulp fluid: a discussion of its significance in reaction to dentinal sensitivity. Caries Res 1 , 310-317.

4. Parshley DH (1986) Dentin permeability, dentin sensitivity, 
and treatment through tubule occlusion. J Endod 12, 465-474.

5. Rimondini L, Baroni C, Carrassi A (1995) Ultrastructure of hypersensitive and non-sensitive dentine. a study on replica models. J Clin Periodontol 22, 212-218.

6. West NX, Lussi A, Seong J, Hellwig E (2013) Dentin hypersensitivity: pain mechanisms and aetiology of exposed cervical dentin. Clin Oral Investig 17, S9-19.

7. Pashley DH, Matthews WG, Zhang Y, Johnson M (1996) Fluid shifts across human dentine in vitro in response to hydrodynamic stimuli. Arch Oral Biol 41, 1065-1072.

8. Orchardson R, Gillam DG (2006) Managing dentin hypersensitivity. J Am Dent Assoc 137, 990-998.

9. Shiau HJ (2012) Dentin hypersensitivity. J Evid Based Dent Pract 12, 220-228.

10. Schmiidlin PR, Sahrmann P (2013) Current management of dentin hypersensitivity. Clin Oral Invetig 17, S55-59.

11. He T, Sun L, Li S, Ji N (2010) The anti-plaque efficacy of a novel stannous-containing sodium fluoride dentifrice: a randomized and controlled clinical trial. Am J Dent $23 \mathrm{Spec}$ Issue $\mathrm{B}, 11 \mathrm{~B}-16 \mathrm{~B}$.

12. Hara AT, Lippert F, Zero DT (2013) Interplay between experimental dental pellicles and stannous-containing toothpaste on dental abrasion. Cries Res 47, 325-329.

13. Passos VF, de Vasconcellos AA, Pequeno PJH, Rodrigues LKA, Santiago SL (2015) Effect of commercial fluoride dentifrices against hydrochloric acid in an erosion-abrasion model. Clin Oral Investig 19, 71-76.

14. João-Souza, Bezerra SJC, de Freitas PM, de Lima NB, Aranha ACC, Scaramucci T (2015) In situ evaluation of fluoride-, stannous- and polyphosphate-containing solutions against enamel erosion. J Dent 63, 30-35.

15. Feng X, Chen X, Cheng R, Sun L, Zhang Y, He T (2010) Breath malodor reduction with use of a stannous-containing sodium fluoride dentifrice: a meta-analysis of four randomized and controlled clinical trials. Am J Dent 23 Spec Issue B, 27B-31B.

16. Ni LX, He T, Chang A, Sun L (2010) The desensitizing efficacy of a novel stannous-containing sodium fluoride dentifrice: an 8-week randomized and controlled clinical trial. Am J Dent 23 Spec Issue B, 17B-21B.

17. Chaknis P, Panagakos FS, DeVizio W, Sowinski J, Petrone D, Proskin H (2011) Assessment of hypersensitivity reduction of a dentifrice containing $0.3 \%$ triclosan, $2.0 \%$ PVM/ MA copolymer, $0.243 \% \mathrm{NaF}$ and specially-designed silica as compared to a dentifrice containing $0.454 \%$ stannous fluoride, sodium hexametaphosphate and zinc lactate and to a dentifrice containing $0.243 \% \mathrm{NaF}$ on dentin hypersensitivity reduction: an 8-week study. Am J Dent 24 Spec Issue A, 14A-20.

18. He T, Chang J, Cheng R, Li X, Sun L, Biesbrock AR (2011) Clinical evaluation of the fast onset and sustained sensitivity relief of a $0.454 \%$ stannous fluoride dentifrice compared to an $8.0 \%$ arginine-calcium carbonate-sodium monofluorophosphate dentifrice. Am J Dent 24, 336-340.

19. Parkinson C, Hughes N, Jeffery P, Jain R, Kennedy L, Qaqish
J et al (2013) The efficacy of an experimental dentifrice containing $0.454 \% \mathrm{w} / \mathrm{w}$ stannous fluoride in providing relief from the pain of dentin hypersensitivity: an 8-week clinical study. Am J Dent 26 Spec Issue A, 25A-31.

20. He T, Barker ML, Biesbrock A, Sharma N (2014) A randomized controlled clinical trial to assess the desensitizing effect of a stannous fluoride dentifrice. Am J Dent 27, 106-110.

21. Parkinson C, Jeffery P, Milleman JL, Milleman KR, Mason S (2015) Confirmation of efficacy in providing relief from the pain of dentin hypersensitivity of an anhydrous dentifrice containing $0.454 \%$ with or without stannous fluoride in an 8-week randomized clinical trial. Am J Dent 28, 190-196.

22. Parkinson C, Hughes N, Hall C, Whelton H, Gallob JT, Mason S (2016) The randomized clinical trials to assess the short-term efficacy of anhydrous $0.454 \% \mathrm{w} / \mathrm{w}$ stannous fluoride dentifrices for the relief of dentin hypersensitivity. Am J Dent 29, 25-32.

23. Miller S, Troung T, Heu R, Stranick M, Bouchard D, Gaffar A (1994) Recent advances in stannous fluoride technology: antibacterial efficacy and mechanism of action towards hypersensitivity. Int Dent J 44, 99-105.

24. Hosoya Y, Tadokoro K, Inoue T, Miyazaki M, Tay FR (2013) Effect of SI-R20401 to remineralize artificial incipient enamel lesions in primary teeth. J Oral Sci 55, 301-310.

25. He T, Britt M, Biesbrock AR (2010) Innovations in global dentifrice technology: an advanced stannous-containing sodium fluoride dentifrice. Am J Dent 23 Spec Issue B, 3B-10.

26. Burnet GR, Wilson RJ, Lucas RA (2013) In vitro studies investigating the dentin tubule-occlusion properties of an experimental anhydrous stannous fluoride dentifrice. Am J Dent 26 Spec Issue A, 10A-14.

27. Endo H, Kawamoto R, Takahashi F, Takenaka H, Yoshida F, Nojiri K et al. (2013) Evaluation of a calcium phosphate desensitizer using an ultrasonic device. Dent Mater J 32, 456-461.

28. Otuyemi OD, Noar JH (1996) A comparison of crown size dimensions of the permanent teeth in a Nigerian and a British population. Eur J Orthod 18, 623-628.

29. Whittaker DK, Barkri MM (1996) Racial variations in the extent of tooth root translucency in ageing individuals. Arch Oral Biol 41, 15-19.

30. Smith TM, Olejniczak AJ, Reid DJ, Ferrell RJ, Hublin JJ (2006) Modern human molar enamel thickness and enameldentine junction shape. Arch Oral Biol 51, 974-995.

31. Brook AH, Griffin RC, Townsend G, Levisianos Y, Russell J, Smith RN (2009) Variability and patterning in permanent tooth size of four human ethnic groups. Arch Oral Biol 54, S79-85.

32. Ohtani S, Yamamoto T (2011) Comparison of age estimation in Japanese and Scandinavian teeth using amino acid racemization. J Forensic Sci 56, 244-247.

33. García-Godoy F (2010) An advanced stannous-containing sodium fluoride dentifrice. Am J Dent 23, 2B. 